\title{
The sense of smell in chronic rhinosinusitis - literature review
}

\author{
Corresponding author: \\ Łukasz Kluczyński \\ Sensory Organs Department, \\ Collegium Medicum, Nicolaus Coper- \\ nicus University, \\ Jagiellońska 13-15 St. \\ 85-067 Bydgoszcz, Poland \\ tel.: (52) 58547 10, fax: (52) 5854035 \\ e-mail: luklu@interia.pl \\ Medical Research Journal 2018; \\ Volume 3, Number 1, 1-9 \\ 10.5603/MRJ.2018.0001 \\ Copyright (C) 2018 Via Medica \\ ISSN 2451-2591
}

\begin{abstract}
SUMMARY
Chronic rhinosinusitis (CRS) is an inflammation of the mucous membrane of the nose and paranasal sinuses. One of the four cardinal symptoms is smell distortion. It is estimated that CRS is the cause of $14-30 \%$ of all olfactory disorders among humans. A wide variety of methods checking olfactory functions have been developed. This literature review is meant to discuss all known up-to-date tests and show their usefulness. The authors also compare the results of treatment, according to the level of smell disorders, in connection with the presence of polyps, time of healing of post-operative wounds, time of the procedure, and others. In conclusion, one can say that all of these characteristics are important and that treatment of dysosmia is a challenging part of chronic sinusitis therapy.

Key words: chronic rhinosinusitis, olfaction test, olfaction disorder, endoscopic sinus surgery, quality of life, Lund Mackay scale, Lund Kennedy scale.
\end{abstract}

Med Res J 2018; 3 (1): 1-9

\section{Introduction}

Chronic rhinosinusitis (CRS) is inflammation of the mucous membrane of the nose and paranasal sinuses lasting for at least 12 weeks [1]. According to the current state of knowledge, CRS is caused by impaired drainage and ventilation of paranasal sinuses, which result from obstruction of the natural ostia. The predisposing factors are: abnormal anatomy, infections, allergies, impaired cilia mobility, laryngopharyngeal reflux, nodules, foreign bodies, a history of injuries, and hormonal and immunological disorders [2]. According to the latest estimates, the prevalence of CRS is from $5.5 \%$ to $10.9 \%$ [3-6]. In order to diagnose CRS as prescribed in the European Position Paper on Rhinosinusitis and Nasal Polyps (EPOS), at least two of four cardinal symptoms have to be diagnosed. At least one of two cardinal symptoms has to be present: either nasal discharge or nasal obstruction. The two remaining symptoms are smell distortion (dysosmia) and facial pain/pressure. Finding nasal polyps, presence of mucopurulent discharge, swelling in the middle nasal meatus, or obstruction found in physical examination, help to diagnose the CRS. In addition, CRS can be diagnosed upon finding inflammatory lesions of the mucous membrane in the ostiomeatal complex in a computed tomography (CT) scan. According to the EPOS, CRS with nasal polyps (CRSwNP) can be distinguished; it is diagnosed when polyps are present on both sides of the middle nasal meatus. They constitute less than $18 \%$ of all cases of CRS [3]. In other cases, CRS without nasal polyps (CRSsNP) is diagnosed [1].

The diagnostic methods commonly used to evaluate the severity of CRS at the local level are:

- Computed Tomography (CT) with the use of the Lund Mackay scoring system. Each of the paranasal sinuses and ostiomeatal complexes is evaluated; each individual anatomical structure is assigned a score of $0-2$. Each side is evaluated separately. A total score of 24 can be assigned [7-8].

- Nasal endoscopy with the use of the Lund Kennedy scoring system. Evaluation includes the presence of polyps in the middle nasal meatus as well as discharge, swelling of the mucous membrane, scars/adhesions, and crusts in the nasal cavities [7-8]. Each of the identified factors is assigned a score of $0-2$, separately for each of the sides. A total score of 20 can be assigned [7-8].

CRS decreases the quality of life and effectiveness of work [9]. Therefore, in view of the epidemiological data provided above, CRS is a serious health concern that requires effective treatment. The treatment of choice for CRS is pharmacological treatment; its specific course depends on the form and severity of the disease evaluated using a Visual Analogue Scale (VAS) and endoscopy. VAS is based on the patient self-assessing 
the severity of symptoms of CRS. An analogue scale of $0-10$ is used, where the highest score indicated by the patient corresponds with the most severe symptoms of CRS.

Treatment of CRS begins with topical steroid treatment. In the case of CRSsNP, irrigation is additionally introduced. In the event of no improvement after three months or a more severe course of the disease identified based on the VAS or endoscopy, antibiotic therapy, as an extension of treatment, should be considered. If pharmacological treatment is not effective, surgical treatment should be considered. However, after surgical treatment, pharmacological treatment, i.e. irrigation and topical steroid therapy, and possibly general steroid and antibiotic therapy, should be continued [1].

Nowadays, the standard of surgical treatment of CRS is Functional Endoscopic Sinus Surgery (FESS). This procedure is based on eliminating the cause behind the CRS by restoring correct ventilation and drainage of the sinuses. So far, surgical intervention within the area of the maxillary sinus is limited to nose side wall structures, i.e. resection of the uncinate process and, if there is a need to gain improved access to the maxillary sinus, widening of its natural ostium. Frontal sinus surgery is, in most cases, limited to intervention within the area of the frontal recess. In the abovementioned cases of sinusitis or if inflammatory lesions occur in anterior ethmoid sinuses, the ethmoid cells are opened. Sphenoid sinusitis is usually treated by widening natural ostia of these sinuses. If ostia of the sinuses are hard to identify, artificial ostia are created. In the case of sphenoid sinusitis or posterior ethmoid sinusitis, posterior ethmoid cells have to be opened. According to different methods of approaching the frontal sinus, three basic types of procedure are identified: Draf I, Draf II a, Draf II b, and Draf III. The technique to be selected depends on the size of the inflammatory lesions, the need to remove lesions in the lumen of the sinus, and anatomical conditions. The Draf I procedure involves draining the sinus without damaging its mucous membrane by removing a fragment of the uncinate process. In the Draf II a procedure, the sinus is approached by resection of bone trabeculae in the vicinity of its ostium and widening of the sinus ostium from the orbital plate to the vertical plate of the middle nasal concha. The Draf II b procedure is based on complete removal of the bottom wall of the frontal sinus between the orbital plate and the nasal septum, including partial removal of the vertical plate of the middle nasal concha in its anterior segment. The most invasive approach to the frontal sinus is gained by using the Draf III technique, also called the endoscopic modified Lothrop procedure. This procedure is initiated by creating a Draf II b-type two-way approach and then resecting the upper anterior part of the nasal septum and the partition inside the frontal sinus. [2].

\section{Examining olfactory dysfunctions in chronic rhinosinusitis}

Olfactory dysfunction is diagnosed in $60-83 \%$ of cases of CRS $[3,7,10-12]$. It is estimated that CRS is the cause of $14-30 \%$ of all olfactory disorders and is the second most common cause of these disorders, the first being acute viral rhinitis and rhinosinusitis $[5,7,13]$. The pathophysiological basis of the olfactory dysfunction is the impairment of nose patency and inflammatory damage to the olfactory epithelium of the nasal cavities [10]. The most common olfactory disorders include: anosmia - loss of the sense of smell, and hyposmia - partial impairment of the sense of smell. Other forms of improper function of the olfactory system, such as hyperosmia - olfactory hypersensitivity and olfactory hallucinations, i.e. smelling odours different from the real ones, are predominantly associated with psychiatric and neurological disorders related to the central nervous system [2]. It is important to note that the sense of smell, together with the sense of taste, plays an important role in the physiology of nutrition, is responsible for defence mechanisms, and has an effect on the quality of life $[3,12]$. In the case of olfactory disorders, it has been noted that the prevalence of psychosocial problems, e.g. depression, is higher when compared to the general population, and the quality of life is decreased $[7,14]$.

A wide variety of methods checking olfactory functions have been developed hitherto; their main aim is to detect quantitative olfactory dysfunctions. They differ in sensitivity, test duration, costs, complexity, the extent to which they are hard to understand for the patient, and type of tested olfactory function. Both objective and subjective examinations are used to evaluate the sense of smell.

Subjective examinations are based on olfactory tests that are commonly used in modern clinical studies. They include:

- Sniffin' Sticks - a test comprised of three parts: olfactory threshold (OT), odour discrimination (OD), and odour identification (OI). The tested person can receive a score of $0-16$ points. The OT test is performed using 16 different concentrations of $\mathrm{n}$-butanol. The tested person, given a set of three sticks - two odourless, one with n-butanol - has to indicate the one with n-butanol. The lowest concentration where the tested person can smell n-butanol is the olfactory threshold. Within a given concentration, n-butanol has to be correctly indicated at least two times. In the OD test, the tested person has to indicate the stick with the odour different from the two other sticks. The test is performed using 16 sets of sticks. In case of the Ol test, the tested person has to choose the correct odour from among the four 
possible answers given. Sixteen odours are tested. The total score that can be given for the entire test is 48 points [3,12,15-16]. Sniffin' Sticks are considered to be a test with the highest sensitivity because they test all of the three functional aspects of the sense of smell (olfactory threshold, odour discrimination, and odour identification). Deviations considered to bear statistical significance in the TDI test are deviations of more than 5.5 points in the follow-up test [3].

- The University of Pennsylvania Smell Identification Test (UPSIT) is the most common and the best available identification test [16]. This test involves identification of 40 odorants on scratched strips by choosing the correct names of smells from among the four possible answers given. This test allows determination of the extent of olfactory damage and detection of malingerers. The probability theory and, to a lesser degree, the use of odour perceived by nerves other than the olfactory nerve, are both used to detect malingerers. By choosing random answers, the persons who truly cannot sense smells will correctly indicate an average of $25 \%$ of odours, scoring 10 out of 40 possible points $[5,12,16]$.

- Cross-Cultural Smell Identification Test (CCSIT), also called the Brief Smell Identification Test (BSIT). This test is also based on odour identification but includes only 12 smells. The odours used are wellknown irrespective of cultural tradition in a given region of the world. Thereby, the probability of indicating a smell incorrectly due to an unfamiliar name of a given substance has been greatly reduced. As it is easy to perform, its cost is low, and its duration is short; the test is used as a screening test.

- Even more simplified identification tests have been described in the literature. In such tests there are only seven smells, separate for each nasal cavity [7,17-18]. The patient has to choose the correct answer after being presented with four possible answers [7,17,-18].

- Butanol Threshold Test (BTT) is a test where the odour detection threshold is tested, the odour is usually $\mathrm{n}$-butanol in nine different concentrations between $0.00061 \%$ and $4 \%$. The patient, being presented with two vials on a random basis (one with $\mathrm{n}$-butanol and one with pure water), has to indicate the one with the stronger smell. The test begins with the lowest concentration of the tested substance. The concentration of $\mathrm{n}$-butanol in each subsequent vial is three times higher than the concentration in the previous one. The odour detection threshold is the lowest concentration at which $n$-butanol can be detected. At least four successive correct answers $[7,12,17,19-20]$ or five correct answers [16] have to be given for a particular concentration. Other odorants, such as peppermint oil, lemon oil, clove oil, or ethyl acetate, can be used in the test as well [17]. The test can be used to diagnose hyposmia [19]. Its advantage is short duration time - usually about five minutes [19]. The sensitivity of the test is comparable to that of CCSIT.

- Self-Administered Odour Questionnaire (SAOQ). In this test, the patient tells whether he/she can smell 20 odours: steamed rice, miso, seaweed, soy sauce, baked bread, butter, curry, garlic, orange, strawberry, green tea, coffee, chocolate, household gas, garbage, timber, excrement, sweat, a flower, and perfume. In case an odour is smelled strongly, a score of 2 is assigned, for faint smell 1 point is assigned, and if no odour is smelled or the odour is unknown, no points are assigned. The result is not taken into account if the patient cannot recognise more than 10 odours [21].

- Intravenous olfaction test: prosultiamine, corresponding to the smell of garlic, or thiamine propyl disulphide, corresponding to the smell of onion, is administered into a forearm vein. A dose of $10 \mathrm{mg}$ of thiamine propyl disulphide is administered within $10 \mathrm{~s}$ [10]. The test outcome is positive if the patient states that he/she can smell a given odour; if not, the outcome is negative.

- Takagi and Toyota (T\&T) olfactometer test - five odours are tested: phenethyl alcohol (rose), methyl cyclopentenolone (caramel), isovaleric acid (sweat), gamma-undecalactone (peach), and skatole (thrash) [10,21].

- Visual Analogue Scale (VAS), mentioned previously, in which the patient self-assesses the sense of smell using an analogue scale, is also in common use, with $0 \mathrm{~mm}$ corresponding to anosmia and $100 \mathrm{~mm}$ corresponding to a perfect sense of smell. The test result is the length from 0 to $100 \mathrm{~mm}$ on the scale. The test is solely based on subjective assessment by the patient, without the use of any odorants $[16,20]$. The second group of olfactory tests are objective examinations where magnetic resonance imaging (MRI), positron emission tomography (PET), olfactory event-related potentials (OERPs), and olfactory ensheathing glial cells (OEGs) are used [22]. However, due to the high cost and limited availability of these tests, objective olfaction assessment methods are used only occasionally.

To evaluate the impact of sense of smell disturbances on quality of life there are several questionnaires, the most popular are described as follows: The Sinonasal Outcome Test (SNOT-22) is comprised of 22 questions evaluated according to a Likert-type scale of $0-5$, where 0 is indicative of absence of the symptom, and 5 means that the symptom is very onerous. A total score of 110 can be achieved. Less detailed questionnaires including 20 questions (SNOT-20) are sometimes used. 
The SNOT-22 questionnaire is used to evaluate the four cardinal symptoms of CRS. Question 6 asks about evaluate facial pain/pressure, question 10 - nasal blockage, question 21 - function of the sense of smell and taste, and question 22 runny nose. [18,23]

Rhinosinusitis Disability Index (RSDI) - includes a total of 30 statements evaluated according to a Likerttype scale of $0-4$, where 0 means 'never' and 4 means 'always'. A total score of 120 can be achieved. Eleven statements are used to evaluate the physical condition, nine statements - the functional condition, and ten statements - the emotional condition. From the viewpoint of evaluating main symptoms of CRS, the most significant questions are: no. 1 - 'The pain or pressure in my face makes it difficult for me to concentrate', no. 6 - 'I am inconvenienced by my chronic runny nose', no. 7 - 'Food does not taste good because of my change in smell', and no. 10 - 'I have difficulty with exertion due to my nasal obstruction' [18,23].

Questionnaire of Olfactory Disorder (QOD) - the test is comprised of three modules:

Questionnaire of Olfactory Disorder - negative statements (QOD - NS) — includes 17 negative statements. Each of them is assigned a score of $0-3$ by the tested person. A total score of 51 can be achieved. Higher score is equivalent to better quality of life. This module is used to evaluate the extent to which dysosmia affects everyday life. The two other modules: Questionnaire of Olfactory Disorder — positive statements (QOD - PS) - two statements, and Questionnaire of Olfactory Disorder - socially desired statements (QOD - SD) - six statements; these last two modules are both of lesser significance as far as evaluation of the quality of life is concerned [23-25].

\section{The effect of the polyps on the sense of smell}

In recent years there have been many original articles published focusing on the comparison of the effect of treatment of CRS versus conservative therapy on improvement in olfactory function and the associated improvement in the patient's quality of life. In the overwhelming majority of clinical studies, patients were qualified according to EPOS 2009- and EPOS 2012-based criteria. The patients were usually divided into two groups, following the abovementioned guidelines, depending on the presence of nasal polyps, as: CRSwNP and CRSsNP.

One study including 113 patients showed that, within the period of six months following FESS surgery, greater improvement in the sense of smell was achieved by patients from the CRSwNP group as compared to those from the CRSsNP group. Improvement in the sense of smell based on the UPSIT test was found in $72.5 \%$ of patients from the CRSwNP group, aggravation in 13.7\% of patients, and no change in $13.7 \%$ of patients [5]. The results provided above suggest that the presence of nasal polyps, obstruction of nasal meatuses by these polyps, and subsequent reduced inflow of odorants to the olfactory epithelium are all of fundamental significance in the pathophysiology of dysosmia. These conclusions were confirmed in a subsequent study where the patients were divided into two groups: group $A$, where the polyps were surgically removed, and group $B$, where the polyps were left intact. In group $A$, after six months of FESS, a statistically significant improvement of UPSIT, SNOT-20, and VAS results was found, whereas in group $B$ significant improvement was found only in the VAS test. It was thereby confirmed that surgical removal of nasal polyps has an effect on improvement of the sense of smell [14]. The same conclusions were reached in a study covering 342 patients, in which improvement of the sense of smell was greater in patients with CRSwNP than in patients with CRSsNP. CT scans were assessed according to the Lund-Mackay scoring system, nasal endoscopy results - according to the Lund-Kennedy scoring system, and the SNOT-22 questionnaire was evaluated, all prior to the surgery and six months after the surgery. Surprisingly, in those patients with the best improvement in all four cardinal symptoms of CRS, the level of the sense of smell improvement was the lowest. It might be caused by the fact that only a small number of patients had been aware of olfactory dysfunctions [26]. It was reported by Kim et al. that only $58 \%$ of patients were aware of dysosmia before smell tests [19]. Similar conclusions were drawn based on observations made six months after FESS surgery. In this specific case, the sense of smell was tested using the TDI test in patients treated for CRSwNP. The observed improvement in the sense of smell was worse than that found in the previous studies, and was seen in $42 \%$ of cases, with the treatment having no impact on the sense of smell observed in $53 \%$ of the subjects, and the sense of smell being aggravated in $5 \%$ of the subjects [3].

To evaluate how age affects the main symptoms of CRS, three age groups were identified among persons suffering from this disease. It was found that in the group of patients below 40 years of age dysosmia is the most uncommon - affecting $37.0 \%$ of the studied group. At the same time, the CRSsNP variant was found to prevail in this group of patients. On the other hand, CRSwNP is more common in the 40-59-year-old age group and in patients older than 60 years. In addition, the percentage of dysosmias increases with age: it is equal to $49.8 \%$ and $53.1 \%$ for the middle-aged group and the oldest age group, respectively, which constitutes yet another confirmation of the fact that the presence of nasal polyps affects the condition of the sense of smell [27]. 
Table 1. CT score by Lund Mackay scale [8]

\begin{tabular}{lccc}
\hline $\begin{array}{l}\text { Scoring } \\
\text { Structure }\end{array}$ & $\mathbf{0}$ & $\mathbf{1}$ & $\mathbf{2}$ \\
\hline Maxillary sinus & no abnormalities or absent & partial opacification & total opacification \\
Frontal sinus & & & \\
Anterior ethmoidal sinus & & \\
Posteriori ethmoidal sinus & & & \\
Sphenoidal sinus & non-occluded & occluded \\
Ostiomeatal complexes & & & \\
\hline
\end{tabular}

Table 2. Nasal endoscopy score by Lund Kennedy scale [8]

\begin{tabular}{lccc}
\hline $\begin{array}{l}\text { Scoring } \\
\text { Appearances }\end{array}$ & $\mathbf{0}$ & $\mathbf{1}$ & $\mathbf{2}$ \\
\hline $\begin{array}{l}\text { Polyp } \\
\text { Discharge }\end{array}$ & absent & polyps in middle meatus only & polyps beyond middle meatus \\
Oedema & & clear, thin & thick, purulent \\
Scarring & & mild & severe \\
Crusting & & & \\
\hline
\end{tabular}

Table 3. Interpretation of olfaction test results

\begin{tabular}{|c|c|c|c|c|c|c|c|c|}
\hline \multicolumn{2}{|l|}{$\begin{array}{l}\text { Research } \\
\text { Olfaction } \\
\text { condition }\end{array}$} & \multirow{2}{*}{$\begin{array}{c}\text { TDI [25] } \\
0-15\end{array}$} & $\begin{array}{c}\text { UPSIT } \\
\text { [16] }\end{array}$ & \multirow{2}{*}{$\begin{array}{c}\text { CCSIT [13] } \\
0-3\end{array}$} & \multirow{2}{*}{$\begin{array}{c}\text { BSIT } \\
0-4[19]\end{array}$} & \multirow{2}{*}{$\begin{array}{c}\text { BTT [13] } \\
0-1\end{array}$} & \multicolumn{2}{|c|}{$\begin{array}{c}\text { SAOQ [21] olfactometer } \\
\text { T\&T } \\
{[10,21]}\end{array}$} \\
\hline Anosmia & & & \multirow{5}{*}{$\begin{array}{l}\text { depending } \\
\text { on age }\end{array}$} & & & & \multirow{4}{*}{$0-28$} & $5,6-5,8$ \\
\hline \multirow{3}{*}{ Hyposmia } & severe & $16-34,5$ & & $4-5$ & \multirow{3}{*}{ 5-8 [19] } & $2-3$ & & $4,2-5,4$ \\
\hline & moderate & & & $6-8$ & & $4-5$ & & $2,6-4,0$ \\
\hline & mild & & & $9-10$ & & $6-7$ & & $1,2-2,4$ \\
\hline Normosmia & & $35-48$ & & $11-12$ & $\begin{array}{c}9-11 \\
{[18,19,23]}\end{array}$ & $8-9$ & $29-40$ & $0-1,0$ \\
\hline
\end{tabular}

TDI — Sniffin' Sticks assess the olfactory threshold, discrimination, and identification; UPSIT — University of Pennsylvania Smell Identification Test; CCSIT — Cross-Cultural Smell Identification Test; BSIT - Brief Smell Identification Test; BTT — Butanol Threshold Test; SAOQ — SelfAdministrated Odour Questionnaire; T\&T — olfactometer (stands for Takagi and Toyota)

\section{The healing of post-operative wounds and improvement in olfactory function}

Another paper aimed to find factors, other than removal of nasal polyps, that improve the sense of smell in CRSwNP patients. They focused on the local condition after surgical treatment in 50 patients. In order to carry out this evaluation, the patients were divided into two groups: the group in which wound healing was good according to endoscopy result assessment using the Lund Kennedy scoring system, and the group with local postoperative complications. By analysing the endoscopy results and the CT scans according to the Lund Mackay scoring system, and performing BTT and CCSIT tests, greater post-operative improvement in the sense of smell was found in patients with proper wound healing as compared with those with local postoperative complications. In addition, it was noted that in patients who did not show improvement in the sense of smell despite good wound healing, severity of dysosmia before FESS was greater, as well as pre-operative changes seen in the CT scan. It is very likely that the cause is partial or total damage to the olfactory epithelium. It was concluded that pre-operative CT scan analysis according to the Lund Mackay scoring system and post-operative observation of the local condition of the wound (short term - swelling and inflammation, long term - scars, recurrence of polyps) can be used to predict post-operative improvement in the sense of smell [13]. 
The role of CT and MRI scans serving as prognostic indicators of improvement in the sense of smell

In a study by Soler et al., comprising 121 patients, CT scan analysis according to the Lund Mackay scoring system was used, but the UPSIT method of olfactory assessment and the method of QOL (Quality of Life) assessment were modified by using a different questionnaire - the QOD-NS. The post-operative assessment period covered the six months immediately after the procedure. In this case, the CT scan allowed prediction of QOL improvement correlated with the sense of smell by using QOD-NS, with the improvement percentage being 53\%. However, QOD-NS was not correlated with UPSIT, probably due to the fact that odour identification is not the only component of the sense of smell affecting the quality of life [23].

Quite different conclusions were arrived at in a study covering 153 patients suffering from CRS. The results were based on the following tests: endoscopy, UPSIT, and smell self-assessment surveys - before the surgery, 3-6 months after FESS, and 12 months after FESS. The results were compared with CT scans scored using the Lund Mackay system. No correlation between the Lund Mackay scores of the CT scans and improvement in the sense of smell was found [28].

An original clinical study was carried out on a group of 31 patients. The study involved assessment of head MRI scans and TDI tests before FESS and three months after surgery. The aim of the study was to show changes in the white matter of the central nervous system. The hypothesis was based on previous confirmed findings pertaining to volumetric changes in the olfactory bulb and grey matter of the olfactory cortex and to plasticity of these areas associated with improvement in the sense of smell. Before the surgery, $36 \%$ of the patients were anosmic, $45 \%$ were hyperosmic, and $19 \%$ were normosmic, i.e. had normal olfactory function. Average improvement after FESS was $9.06 \%$. The patients were divided into two groups: one with significant olfactory function improvement (38.71\%) and one without significant olfactory function improvement. It was found in the case of the group with significant olfactory function improvement that there is a close correlation with radiographic MRI analysis of structural changes in the left anterior area of the cingulate gyrus and the right amygdala [15].

In order to link instrumental examinations with the achieved results of tests for olfaction and quality of life related thereto, a new endoscopy scale - the "Olfactory Cleft Endoscopy Scale" (OCES) — has been developed [24]. This method is designed for post-operative monitoring of the local condition with the use of the same type of endoscope as the one used during the proce- dure. The evaluation covers five factors, each given a score of 0-2: presence of discharge, swelling, polyps, crusting, and scarring. Each side is evaluated separately. Therefore, the maximum total score is 20 . This scale was validated in a group of 38 patients and was found to correlate with TDI and QOD-NS.

\section{The effect of time after the procedure on the condition of the sense of smell}

The degree of smell impairment before starting therapy, either surgical or conservative, affects prognosis. The more severe the olfactory dysfunction was before surgical treatment, the greater the statistical improvement of the sense of smell as a result of the administered treatment will be. However, in the most extreme cases, i.e. in the group of patients who had been diagnosed with very severe olfactory dysfunction before FESS, even aggravation of olfactory function after the surgery was noted [28].

In a study based on early outcomes achieved one and three months after FESS, a gradual decrease in the percentage of patients with dysosmias, from $70 \%$ before the surgery to $22.5 \%$ one month after the procedure and then to $10 \%$ of subjects within three months after the procedure, was shown by means of BTT. Furthermore, improvements in both the VAS scale results and Lund Mackay endoscopy scoring results were observed [17].

A broader analysis of the prevalence and severity of symptoms was conducted in another study involving 62 patients. Remission of all four cardinal symptoms of CRS was observed in all patients. Improvement in the sense of smell in the period immediately following the procedure was found in $96.3 \%$ of cases. In the same study, follow-up examinations were conducted at 1, 3, 6,12 , and 24 months after FESS. Dysosmia was revealed to be an early sign of recurrence of nasal polyps, whereas nasal obstruction and facial pain were found to be late symptoms of recurrence of nasal polyps [29].

A similar post-operative period was analysed in another study in which two timeframes were taken into account: 6-12 months after the procedure and 1224 months after FESS [30]. Both Lund Mackay-scored CT and nasal endoscopy were conducted before the procedure. It was proven that improvement in the sense of smell can barely be predicted based on pre-operative examination. The abovementioned objective tests were poorly correlated with subjective olfactory tests related to QOL improvement.

A unique study assessed long-term outcomes of CRS treatment in 45 patients. In the study course the olfaction tests were carried out at three months and five years after the FESS surgery. The results were based on VAS, BTT, and smell tests. In the five-year period, 
the sense of smell as measured in the abovementioned tests was improved by $79 \%, 85 \%$, and $53 \%$, respectively. Significant improvement was observed for both early and long-term treatment outcomes. Even though test results five years after the surgery were worse when compared to results after three months, no significant worsening of results between these two periods was found [12].

\section{Comparison of various types of olfactory tests}

A study by Kim et al. aimed to find the best olfactory test methods, as well as the usefulness of BSIT in comparison with BTT. The study was conducted in a group of 68 patients before surgical treatment and six months after treatment by means of the two tests and a VAS scale. The results showed that BSIT can replace BTT. The latter is methodologically better; on the other hand, the advantages of BSIT are shorter duration of the test and the fact that the staff do not need to be provided with special training. Sensitivity of BSIT was deemed to be $63.8 \%$, while its specificity was $93.8 \%$. In addition, in the case of $83.0 \%$ of patients suffering from olfactory dysfunction, $41.2 \%$ achieved improvement in the sense of smell, $25.0 \%$ noted aggravation of this sense, and in $33.8 \%$ the sense of smell remained unchanged [19].

\section{The role of conservative treatment}

In order to optimise post-operative care, the effect of irrigation on olfactory function and the quality of life was studied in 110 patients [31]. Subjects were divided into three groups: the no irrigation group, the water irrigation group, and the ionised acidic water irrigation group (such water shows antibacterial activity). UPSIT and SNOT-20 tests were conducted within each group before FESS and three months after the procedure. Significant improvement in patients' condition was observed in each group, but the improvement was greater in groups where irrigation was used [31].

Budesonide irrigation after FESS has also been studied. For this purpose, patients treated for CRSwNP with FESS were divided into two groups: one treated with sodium chloride $(\mathrm{NaCl})$ irrigations and one treated with $\mathrm{NaCl}$ and budesonide irrigations, and subjected to UPSIT, phenethylamine (PEA) test, SNOT-22, Rhinosinusitis Outcome Measure (RSOM) 31, and the RSDI test four times, including three times after the surgical procedure (1-2 weeks after the procedure, 3-8 weeks after the procedure, and 3-6 months after the procedure). Improvement in the quality of life was found to be similar in the two groups [32].
A significant impact of sense of smell improvement on quality of life and mental health was confirmed. The TDI test, QOD questionnaires, and other mental health assessment scales, including the Beck Depression Inventory, were used for this purpose [25]. In a study employing BSIT for assessment of the sense of smell in 5445 patients, no close correlation with scores recorded in the SNOT and RSDI questionnaires evaluating the quality of life were found. This might be related to the low sensitivity of BSIT associated with its limited capability of differentiating the degree of olfactory dysfunction. This claim is supported by the low percentage of dysosmia recorded in the study, amounting to $28.3 \%$. Nevertheless, the significant correlation between severity of olfactory impairment and CT scan evaluation according to the Lund Mackay system, as well as nasal endoscopy result evaluation according to the Lund Kennedy system, was still confirmed [18].

Even if primary pharmacological treatment is unsuccessful, not all patients are chosen to undergo surgical treatment, regardless of indications for the procedure. The impact of surgical treatment on the sense of smell was evaluated by comparing the group of patients who had undergone surgical treatment with the group of patients who had continued their pharmacological treatment. Primary pharmacological treatment was associated with no effect in both groups. The first group covered $80 \%$ of patients, while the second group covered $20 \%$. BSIT, RSDI, and SNOT-22 tests were repeated after six months; in the case of the surgical treatment group, the first tests were performed before FESS. A total of 280 patients were tested. As far as the first group is concerned, improvement in the sense of smell was observed in 66 patients (41.2\%). As for the second group, improvement was found in 17 cases (37.9\%). Comparable improvement was shown in both groups irrespective of the presence of polyps. As regards the patients diagnosed with CRSwNP, correlation between olfactory improvement and nose patency improvement was shown, whereas in case of the patients diagnosed with CRSsNP, correlation between olfactory improvement and reduction of nasal discharge was shown. Patients with normosmia were found not to suffer from olfactory impairment after treatment [23].

\section{Eosinophilic Rhinosinusitis}

A somewhat different method of CRS classification has been developed in Japan in the Japanese Epidemiological Survey of Refractory Eosinophilic Chronic Rhinosinusitis Study (JESREC study). CRS is classified as eosinophilic chronic rhinosinusitis (ECRS) on the basis of assessment regarding the presence of: nasal obstruction with dysosmia (score 0-3), polyps on both sides of the middle nasal meatus (score 0-2), eosino- 
phils (score 0-10), and ethmoid cells bilateral shadow in CT (score 0-2). A score of 11 or higher is equivalent to diagnosis of ECRS, while a score of less than 11 means non-eosinophilic chronic rhinosinusitis (NECRS) $[10,21]$.

The classification system presented above was applied to 57 patients tested by means of SNOT-22, Patient-Reported Outcomes Measurement Information System (PROMIS) 29, and quality of life (QOL) questionnaires [33]. The discussed study proved that patients suffering from ECRS experience more onerous smell and taste impairments, nasal obstruction, and nasal congestion than those suffering from NECRS. Among the group of 27 patients with ECRS, 21 were diagnosed with CRSwNP, while the rest, with CRSsNP, according to the EPOS criteria.

Another Japanese study employed the intravenous olfaction test and T\&T olfactometer testing [20]. In a group of 272 patients, 193 were classified as ECRS patients and 79 as NECRS patients. Correlation between occurrence of dysosmia defined by certain olfactory threshold values and results of CT scanning defined by nose and paranasal sinuses shadowing were found. In the group of patients suffering from ECRS, dysosmia is more severe and takes the form of anosmia, while in the case of NECRS, hyposmia is dominant [10]. Another study covering a group of 89 patients and a longer post-operative period, with patients divided into ECRS-diagnosed and NECRS-diagnosed patients, was conducted. In this study, the SAOQ test as well as VAS and T\&T tests were performed before FESS and $3,6,12$, and 24 months after the procedure. Average improvement in the sense of smell was achieved within six months of the procedure (including $50-60 \%$ of anosmic patients noticing improvement within half a year). It was shown that eosinophilia is a predisposing factor of olfactory dysfunction because eosinophilic infiltration in the olfactory epithelium leads to sensory impairment. In cases where eosinophilia is severe, one has to expect dysosmias to recur.

\section{Other factors affecting the sense of smell}

As tobacco smokers have a poorer sense of smell, the researchers decided to analyse the impact of smoking on improvement in the quality of life after surgical treatment (FESS). A group of 111 patients took part in the study; their CT scans were analysed according to the Lund Mackay scoring system, and their nasal endoscopy results - according to the Lund Kennedy system; they were also subjected to an olfactory test and filled in the QOD questionnaire. All examinations were performed before the procedure and 12 months after the procedure [34]. It was found that improvement in the quality of life was present in both smokers and non-smokers, but the degree of improvement was much better in non-smokers.

It was demonstrated that aspirin sensitivity or lack of aspirin sensitivity had no impact on olfactory dysfunction in CRS [35].

\section{Conclusions}

1. The extent of changes in chronic rhinosinusitis with polyps is detrimental to olfactory function.

2. Dysosmia is the least onerous of the four cardinal symptoms.

3. Improvement in the sense of smell is correlated with proper surgical wound healing.

4. Irrigation can be used as a useful element of olfactory organ treatment, with pharmacological treatment and surgical treatment having a similar effect.

5. Prognosis of improvement in the sense of smell is worse in ECRS.

6. Tobacco smoking also aggravates the prognosis of improvement in olfactory function.

\section{References}

1. Fokkens WJ, Lund VJ, Mullol J, et al. EPOS 2012: European position paper on rhinosinusitis and nasal polyps 2012. A summary for otorhinolaryngologists. Rhinology. 2012; 50(1): 1-12, doi: 10.4193/Rhino50E2, indexed in Pubmed: 22469599.

2. Niemczyk K, Jurkiewicz D, Składzień J, et al. Otorynolaryngologia kliniczna, Warsaw: MediPage; 2014; 1:338 - 350; 2:321. ; 323: 342.

3. Walliczek-Dworschak U, Pellegrino R, Taube F, et al. Chemosensory function before and after multimodal treatment in chronic rhinosinusitis patients. Laryngoscope. 2018; 128(3): E86-E90, doi: 10.1002/lary.26873, indexed in Pubmed: 28895150.

4. Hirsch AG, Stewart WF, Sundaresan AS, et al. Nasal and sinus symptoms and chronic rhinosinusitis in a population-based sample. Allergy. 2017; 72(2): 274-281, doi: 10.1111/all.13042, indexed in Pubmed: 27590749

5. Andrews PJ, Poirrier AL, Lund VJ, et al. Outcomes in endoscopic sinus surgery: olfaction, nose scale and quality of life in a prospective cohort study. Clin Otolaryngol. 2016; 41(6): 798-803, doi: 10.1111/coa.12665, indexed in Pubmed: 27120034

6. Lind $\mathrm{H}$, Joergensen $\mathrm{G}$, Lange $\mathrm{B}$, et al. Efficacy of ESS in chronic rhinosinusitis with and without nasal polyposis: a Danish cohort study. Eur Arch Otorhinolaryngol. 2016; 273(4): 911-919, doi: 10.1007/s00405015-3667-9, indexed in Pubmed: 26031891

7. Gupta D, Gulati A, Singh I, et al. Endoscopic, radiological, and symptom correlation of olfactory dysfunction in pre- and postsurgical patients of chronic rhinosinusitis. Chem Senses. 2014; 39(8): 705-710, doi: 10.1093/chemse/bju042, indexed in Pubmed: 25165069.

8. Lund VJ, Kennedy DW. Staging for rhinosinusitis. Otolaryngol Head Neck Surg. 1997; 117(3 Pt 2): S35-S40, doi: 10.1016/S019459989770005-6, indexed in Pubmed: 9334786.

9. Rudmik L, Smith KA, Kilty S. Endoscopic polypectomy in the clinic: a pilot cost-effectiveness analysis. Clin Otolaryngol. 2016; 41(2): 110-117, doi: 10.1111/coa.12473, indexed in Pubmed: 26053107.

10. Saito T, Tsuzuki K, Yukitatsu Y, et al. Correlation between olfactory acuity and sinonasal radiological findings in adult patients with chronic rhinosinusitis. Auris Nasus Larynx. 2016; 43(4): 422-428, doi: 10.1016/j. anl.2015.12.007, indexed in Pubmed: 26743838.

11. Minwegen F, Thomas JP, Bernal-Sprekelsen M, et al. Predictive value of disease severity on self-reported rating and quantitative measures of olfactory function outcomes after primary endoscopic sinus surgery. A prospective study. Rhinology. 2014; 52(4): 437-443, doi: 10.4193/Rhin14.043, indexed in Pubmed: 25479229 
12. Rudmik L, Smith TL. Olfactory improvement after endoscopic sinus surgery. Curr Opin Otolaryngol Head Neck Surg. 2012; 20(1): 29-32, doi: 10.1097/MOO.0b013e32834dfb3d, indexed in Pubmed: 22143338.

13. Kim DW, Kim JY, Kim SW, et al. Postoperative olfactory results in chronic rhinosinusitis with nasal polyposis according to wound healing status. Clin Exp Otorhinolaryngol. 2013; 6(3): 146-151, doi: 10.3342/ceo.2013.6.3.146, indexed in Pubmed: 24069517.

14. Kuperan AB, Lieberman SM, Jourdy DN, et al. The effect of endoscopic olfactory cleft polyp removal on olfaction. Am J Rhinol Allergy. 2015; 29(4): 309-313, doi: 10.2500/ajra.2015.29.4191, indexed in Pubmed: 26163252

15. Güllmar D, Seeliger T, Gudziol H, et al. Improvement of olfactory function after sinus surgery correlates with white matter properties measured by diffusion tensor imaging. Neuroscience. 2017; 360: 190-196, doi: 10.1016/j.neuroscience.2017.07.070, indexed in Pubmed: 28797663.

16. Thompson CF, Kern RC, Conley DB. Olfaction in Endoscopic Sinus and Skull Base Surgery. Otolaryngol Clin North Am. 2015; 48(5): 795-804, doi: 10.1016/i.otc.2015.05.007, indexed in Pubmed: 26117298.

17. Gupta D, Gulati A, Singh I, et al. Impact of endoscopic sinus surgery on olfaction and use of alternative components in odor threshold measurement. Am J Rhinol Allergy. 2015; 29(4): e117-e120, doi: 10.2500/ajra.2015.29.4207, indexed in Pubmed: 26163240.

18. Alt JA, Mace JC, Buniel MCF, et al. Predictors of olfactory dysfunction in rhinosinusitis using the brief smell identification test. Laryngoscope. 2014; 124(7): E259-E266, doi: 10.1002/lary.24587, indexed in Pubmed: 24402746 .

19. Kim BG, Oh JH, Choi HaNa, et al. Simple assessment of olfaction in patients with chronic rhinosinusitis. Acta Otolaryngol. 2015; 135(3): 258-263, doi: 10.3109/00016489.2014.974288, indexed in Pubmed: 25625195

20. Briner HR, Jones N, Simmen D. Olfaction after endoscopic sinus surgery: long-term results. Rhinology. 2012; 50(2): 178-184, doi: 10.4193/Rhino11.213, indexed in Pubmed: 22616079.

21. Oka H, Tsuzuki K, Takebayashi $\mathrm{H}$, et al. Olfactory changes after endoscopic sinus surgery in patients with chronic rhinosinusitis. Auris Nasus Larynx. 2013; 40(5): 452-457, doi: 10.1016/j.anl.2012.12.001, indexed in Pubmed: 23312885.

22. Dżaman K. Dżaman K, Współczesne metody badania węchu i smaku, Otorynolaryngologia. 2008; 7: 173

23. Soler ZM, Smith TL, Alt JA et al. Olfactory-specific quality of life outcomes after endoscopic sinus surgery. Int Forum Allergy Rhinol. 2016; 6(4): 407-413, doi: 10.1002/alr.21679, indexed in Pubmed: 26678351

24. Soler ZM, Hyer JM, Karnezis TT, et al. The Olfactory Cleft Endoscopy Scale correlates with olfactory metrics in patients with chronic rhinosinusi- tis. Int Forum Allergy Rhinol. 2016; 6(3): 293-298, doi: 10.1002/alr.21655, indexed in Pubmed: 26718315

25. Katotomichelakis M, Simopoulos E, Tripsianis G, et al. Improvement of olfactory function for quality of life recovery. Laryngoscope. 2013; 123(11): E10-E16, doi: 10.1002/lary.24113, indexed in Pubmed: 23686475.

26. DeConde AS, Mace JC, Alt JA, et al. Investigation of change in cardinal symptoms of chronic rhinosinusitis after surgical or ongoing medical management. Int Forum Allergy Rhinol. 2015; 5(1): 36-45, doi: 10.1002/alr.21410, indexed in Pubmed: 25236780

27. Busaba NY. The impact of a patient's age on the clinical presentation of inflammatory paranasal sinus disease. Am J Otolaryngol. 2013; 34(5): 449-453, doi: 10.1016/j.amjoto.2013.03.013, indexed in Pubmed: 23702318.

28. Szaleniec J, Wróbel A, Strek P, et al. Smell impairment in chronic rhinosinusitis - evaluation of endoscopic sinus surgery results and review of literature concerning olfactory function predictors. Otolaryngol Pol. 2015; 69(1): 33-44, doi: 10.5604/00306657.1131143, indexed in Pubmed: 25753166.

29. Bakhshaee M, Sharifian MR, Ghazizadeh AH, et al. Smell Decline as a good Predictor of Sinonasal Polyposis Recurrence after Endoscopic Surgery. Iran J Otorhinolaryngol. 2016; 28(85): 125-134, indexed in Pubmed: 27280099.

30. Hsu CY, Wang YP, Shen PH, et al. Objective olfactory outcomes after revision endoscopic sinus surgery. Am J Rhinol Allergy. 2013; 27(4): e96100, doi: 10.2500/ajra.2013.27.3939, indexed in Pubmed: 23883800.

31. Jiang RS, Liang KL, Wu SH, et al. Electrolyzed acid water nasal irrigation after functional endoscopic sinus surgery. Am J Rhinol Allergy. 2014; 28(2): 176-181, doi: 10.2500/ajra.2014.28.4015, indexed in Pubmed: 24717957.

32. Rawal RB, Deal AM, Ebert CS, et al. Post-operative budesonide irrigations for patients with polyposis: a blinded, randomized controlled trial. Rhinology. 2015; 53(3): 227-234, doi: 10.4193/Rhin14.196, indexed in Pubmed: 26363164.

33. Thompson CF, Price CPE, Huang JHe, et al. A pilot study of symptom profiles from a polyp vs an eosinophilic-based classification of chronic rhinosinusitis. Int Forum Allergy Rhinol. 2016; 6(5): 500-507, doi: 10.1002/alr.21687, indexed in Pubmed: 26683389.

34. Katotomichelakis M, Simopoulos $E$, Tripsianis $G$, et al. The effects of smoking on quality of life recovery after surgery for chronic rhinosinusitis. Rhinology. 2014; 52(4): 341-347, doi: 10.4193/Rhin, indexed in Pubmed: 25479212

35. Fountain CR, Mudd PA, Ramakrishnan VR et al. Characterization and treatment of patients with chronic rhinosinusitis and nasal polyps. Ann Allergy Asthma Immunol. 2013; 111(5): 337-341, doi: 10.1016/j. anai.2013.07.017, indexed in Pubmed: 24125137. 